
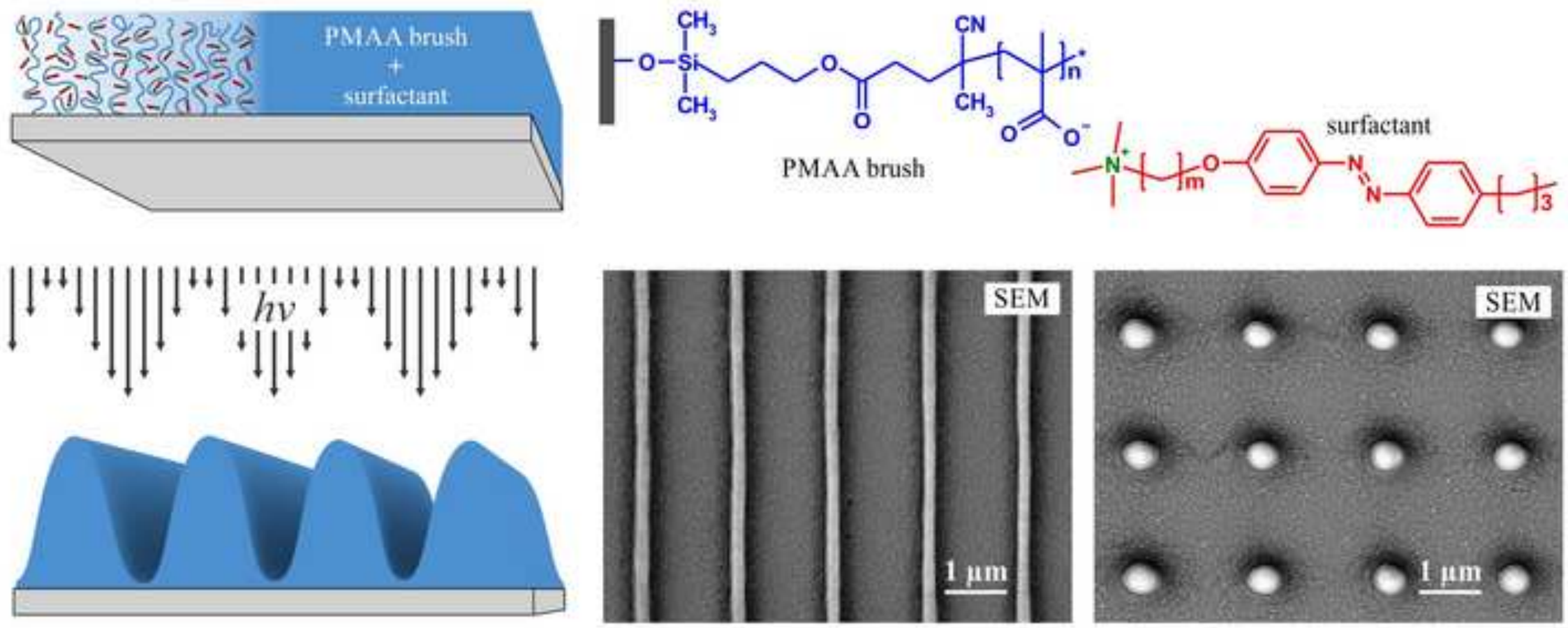

(C) 2015. This manuscript version is made available under the Elsevier user license http://www.elsevier.com/open-access/userlicense/1.0/ 


\title{
Making polymer brush photosensitive with azobenzene containing surfactants
}

\author{
Alexey Kopyshev, ${ }^{1}$ Nino Lomadze, ${ }^{1}$ David Feldmann, ${ }^{1}$ Jan Genzer, ${ }^{2}$ Svetlana Santer ${ }^{1}$ \\ ${ }^{1}$ Institute of Physics and Astronomy, University of Potsdam, 14476 Potsdam, Germany \\ 2 Department of Chemical \& Biomolecular Engineering, North Carolina State University, Raleigh, NC \\ 27695-7905, USA
}

AUTHOR EMAIL ADDRESS: santer@uni-potsdam.de RECEIVED DATE

TITLE RUNNING HEAD: Photosensitive brushes, azobenzene containing cationic surfactants

\section{ABSTRACT}

We report on rendering polyelectrolyte brushes photosensitive by loading them with azobenzenecontaining cationic surfactants. Planar poly(methacrylic acid) (PMAA) brushes are synthesized using the "grafting from" free-radical polymerization scheme followed by exposure to a solution of photosensitive surfactants consisting of positively-charged head groups and hydrophobic tails into which azobenzene moieties are inserted. In this study the length of the hydrophobic methylene spacer connecting the azobenzene and the charged head group ranges from 4 to $10 \mathrm{CH}_{2}$ groups. Under irradiation with UV light, the photo-isomerization of azobenzene integrated into a surfactant results in a change in size, geometry, dipole moment and free volume of the whole molecule. When the brush 
loaded with photosensitive surfactants is exposed to irradiation with UV interference patterns, the topography of the brush deforms following the distribution of the light intensity, exhibiting surface relief gratings (SRG). Since SRG formation is accompanied by a local rupturing of polymer chains in areas from which the polymer material is receding, most of the polymer material is removed from the surface during treatment with good solvent, leaving behind characteristic patterns of lines or dots. The azobenzene molecules still integrated within the polymer film can be removed by washing the brush with water. The remaining nano-structured brush can then be re-used for further functionalization. Although the opto-mechanically induced rupturing occurs for all surfactants, larger species do not penetrate deep enough into the brush such that after rupturing a leftover layer of polymer material remains on the substrate. This indicates that rupturing occurs predominantly in regions of high surfactant density.

KEYWORDS: azobenzene containing cationic surfactants, photosensitive polymer brushes, optomechanically induced scission of polymer chains

\section{Introduction}

Polyelectrolyte brushes consisting of charged polymer chains covalently attached with one end to a solid surface have attracted much theoretical and experimental attention in the last several decades. ${ }^{1}$ This type of soft matter can bear a broad range of physico-chemical responses, ${ }^{2,3,4,5}$ e.g., it can change its shape, thickness, surface energy, or roughness upon variation of external conditions such as $\mathrm{pH}$ and the ionic strength of the solution; ${ }^{6,7,8,9}$ with many conceivable applications; the interest in this material thus is not

only academic. ${ }^{1}$ Additionally, its functionality can further be enhanced by formation of supramolecular complexes between the polyelectrolyte brush and oppositely charged small substances such as 
surfactants, ${ }^{10,11,12}$ proteins, $^{13,14,15}$ nano-particles ${ }^{16,17}$ and synthetic oligomers. ${ }^{18,19}$ For instance, incorporation of palladium nano-particles into spherical polyelectrolyte brushes render them catalytically active. ${ }^{16}$ Due to the high stability and high concentration of reactive centers per unit area of the coating, polymer brushes loaded with proteins are being successfully used for the production of antibody microarrays suitable for decreasing the detection limit. ${ }^{15}$ The decoration of polymer brushes with gold nano-particles provides a way of tuning the optical response from plasmon near fields. ${ }^{20}$

Recently, we have proposed the concept of photosensitive polymer grafts by loading polymer brushes with azobenzene-containing surfactants. ${ }^{21}$ The surfactants consist of a positively charged head group and a hydrophobic tail containing an azobenzene group. Under irradiation the azobenzene molecule undergoes reversible photo-isomerization from a trans- to a cis-conformation accompanied by a change in size, free volume and dipole moment. Typically, the dipole moment increases significantly on isomerizing from trans-to-cis. ${ }^{22}$ When the azobenzene molecule is integrated into the hydrophobic tail of a surfactant molecule, one can easily toggle the hydrophobicity of the surfactant just by alternating irradiation with UV and blue light. ${ }^{23}$ Complex formation between photosensitive surfactants and polyelectrolyte chains (DNA, PAA) or hydrogels allows for light-controlled shape and size of the corresponding soft nano-objects. ${ }^{24,25,26}$ Thus, at certain concentrations of the surfactant molecules, the DNA chain is compacted from an expanded coil to a globular conformation due to screening of the electrostatic repulsions between the charges along the polyelectrolyte on the one hand, and the hydrophobic interactions of the surfactants binding to the DNA on the other. ${ }^{27}$ Under irradiation the compact globuli can unfold since the surfactants become hydrophilic during trans-cis isomerization and consequently retreat from the DNA strand. ${ }^{28,29}$

In addition to rendering single nano-objects photosensitive with azobenzenes, microscopic response of a thin polymer film can also be triggered with integrated azobenzenes. In this case, upon irradiation with an interference pattern, pronounced physical differences between both isomers (trans and cis) result in 
the formation of so-called surface relief gratings (SRG). ${ }^{30,31}$ The photosensitive polymer film, even in the glassy state that has to be assumed under the experimental conditions, deforms following the distribution of the intensity or polarization pattern of the incident light. ${ }^{32,33}$ This phenomenon has been observed both in physisorbed polymer films ${ }^{34,35,36,37}$ and in polymer brushes. ${ }^{38,39,21}$

In our previous study ${ }^{38,39}$ azobenzene-containing surfactants were attached as side groups to the tethered polymer chains, either covalently by a posteriori modification of the polyelectrolyte brush using a special chemical protocol or by simply utilizing ionic interactions between the azobenzene containing cationic surfactants and the poly(methylmethacrylate-b-methacrylic acid) (PMMA-b-PMAA) diblockcopolymer brush. ${ }^{21}$ We have found that local opto-mechanical scission of the polymer chains can be observed exclusively in regions where the polymer material depletes under irradiation with a UV interference pattern. Rupturing then occurs at the interface between the PMMA and PMAA blocks. This observation supports the hypothesis that opto-mechanical stresses generated during deformation of the polymer might be fairly large,,$^{40,41,42,43}$ as the forces required to break a covalent bond are of the order of several nN. ${ }^{44}$

In this paper we study interactions between poly(methacrylic acid) homopolymer polyelectrolyte brushes and cationic azobenzene containing surfactants differing in the length of the hydrophobic spacer connecting the azobenzene group and the charged head group. We have found that the optomechanically induced rupturing of the polymer chains takes place for all surfactants. However, the penetration of the azobenzene modifier into the brush decreases with increasing the size of the azobenzene surfactant, thus altering the extent of the rupturing. 


\section{Experimental Section}

\section{Synthesis of PMAA brushes}

Poly(methacrylic acid) (PMAA) brushes were grown from AMCS (dimethylchlorosilylpropyl 4isobutyronitrile-4-cyano pentanoate) initiator centers immobilized on a silicon wafer or a glass surface in the presence of triethylamine (TEA) in dry toluene solution, by thermal initiation at $60^{\circ} \mathrm{C}$ utilizing surface initiated free radical polymerization. ${ }^{45}$ The reaction conditions give rise to a grafting density of $\Gamma(\mathrm{t})=0.016 \mu \mathrm{mol} / \mathrm{m}^{2}$, i.e. $\sigma=0.01 \mathrm{~nm}^{-2} .{ }^{46}$ Molecular weight of the attached PMAA was determined to be $\mathrm{M}_{\mathrm{n}}=3 \cdot 10^{6}$ Da using an equation: $M_{n}=\frac{\Gamma \cdot h}{\rho}$, where $\mathrm{h}$ is the thickness of the brush measured using AFM scratch experiment, $\rho=1.12 \mathrm{~g} / \mathrm{cm}^{3}$ is density of the PMAA. After polymerization the sample was extracted with DMF in a Soxhlet apparatus for at least 10 hours. This permits to remove all noncovalently attached polymers that are formed in solution. The dry thickness of the brush was $(42 \pm 3)$ nm as determined from AFM "scratching" measurements.

\section{Synthesis and characterization of cationic azobenzene containing surfactants}

Cationic azo-surfactants with different spacers were synthesized by azocoupling p-alkylaniline with phenol, following subsequent reaction with appropriate dibromoalkane and quaternisation with trimethylamine. ${ }^{47}$ The length of the spacer ranges between 4 and 10 methylene $\left(\mathrm{CH}_{2}\right)$ groups $($ Fig. 1b and c). The surfactant was dissolved in water (MilliQ) and kept in the dark for several days to ensure complete relaxation to the trans configuration.

The photoisomerization behavior of the surfactant is described in detail elsewhere. ${ }^{29}$ All surfactants in the dark state (trans conformation) are characterized by an absorption band with a maximum at $353 \mathrm{~nm}$, while the cis isomer is characterized by two absorption bands with maxima at $313 \mathrm{~nm}$ and at $437 \mathrm{~nm}$. 
The band with maximum at $\sim 240 \mathrm{~nm}$ presented in both isomers corresponds to the absorption of the $\pi$ conjugated benzene rings. The lifetime of the cis isomer in the brush is $~ 22$ hours (see Supporting Inofrmation, Fig. S1).

Binding of photosensitive surfactants: PMAA brushes were placed in a surfactant solution of a given concentration for 16 hours at room temperature followed by placing the sample in a water bath for 15 minutes. The surfactant molecules bind ionically and hydrophobically to the brush resulting in an increase of the brush thickness. The degree of binding $(\beta)$ was determined by equation $(1):^{11}$

$$
\beta=\frac{\frac{h_{b}^{a z o}}{h_{b}}-1}{\frac{M_{0}^{a z o+M A A}}{M_{0}^{M A A}}-1}
$$

where $h_{b}$ and $h_{b}^{a z o}$ are the brush dry thicknesses before and after complex formation, respectively. The molecular weight of repeat unit of the pure brush is $M_{0}^{M A A}=86 \mathrm{Da}$, while the molecular weight of the repeat units after complexation with surfactants is $M_{0}^{a z o+M A A}=426,481,509$, and 537 Da for surfactants comprising 4, 6, 8, and $10 \mathrm{CH}_{2}$ spacer units, respectively. In our calculations of the molecular weight of $M A A$ surfactant complex, $M_{0}^{a z o+M A A}$, we do not account for the molar mass of the $\mathrm{Br}^{-}$counter-ion assuming pure ionic interactions between the carboxylic groups and the surfactant molecules. The degree of binding gives the number of surfactant molecules per methacrylic acid (MAA) monomer units in the brush. This quantity can be interpreted as the total absorption of a surfactant into brush.

\section{Methods}

Transmission FT-IR spectra of the attached polymer layers were recorded using a Perkin Elmer FTIRspectrometer Spectrum 2000. FT-IR spectra of the PMAA brushes before and after loading with azobenzene containing surfactant are shown in SP-1 (Supporting Information, Fig. S2). The 
characterization of the surface topography of PMAA brushes was carried out with atomic force microscopy (AFM) (Nanoscope V, Brucker, USA). The microscope was operated in tapping mode, using commercial tips (NanoSensors) with a resonance frequency of $\sim 300 \mathrm{kHz}$, and a spring constant of $\sim 50 \mathrm{~N} / \mathrm{m}$. The AFM micrographs were recorded in air at a temperature of $\sim 23^{\circ} \mathrm{C}$. For thickness measurements, the brushes were scratched to remove the material and the step height between the top of the brush and the carried substrate was measured using the AFM cross-section analysis. All experiments were carried out in a room with yellow light to avoid any premature isomerization of the surfactant.

The Lloyd's mirror scheme with He-Cd laser (Kimmon) operating at $\lambda=325 \mathrm{~nm}$ (total power of $\sim 3 \mathrm{~mW}$ ) was used as UV interference lithography. ${ }^{39}$ The periodicity of the interference pattern is given by $d=\lambda / \sin (\theta / 2)$, where $\lambda$ is the wavelength of the incident light, and $\theta$ is the angle between the incoming laser beam and the mirror surface. Irradiation time of 10 minutes and the periodicity of interference pattern of $1.4 \mu \mathrm{m}$ were kept constant for all experiments.

\section{Results and Discussion}

To render the poly(methacrylic acid) (PMAA) brush photosensitive, azobenzene containing cationic surfactants were added by exposing the brush to surfactant aqueous solution for 16 hours followed by rinsing with pure water, the surfactants are retained within the brush as shown schematically in Fig. 1a. In the following we show results for a polyelectrolyte brush of fixed molecular weight and grafting density of $\mathrm{M}_{\mathrm{n}}=3 \cdot 10^{6} \mathrm{Da}$ and $\sigma=0.01 \mathrm{~nm}^{-2}$, respectively. The dry thickness of the brush is (42 \pm 3$) \mathrm{nm}$ as measured by AFM. In this paper we utilize four surfactants differing in the length of the hydrophobic spacer connecting the charged head group and the azobenzene unit. The space spans a range of $4 \mathrm{CH}_{2}$

(Fig. 1b) up to $10 \mathrm{CH}_{2}$ groups (Fig. 1c). The surfactant with the shortest spacer (i.e., $4 \mathrm{CH}_{2}$ ) also has a shortest length of the alkyl tail (Fig. 1b). 
When the brush is loaded with surfactants, the brush thickness increases; the thickness increase exhibits a characteristic s-shaped curves and it correlates with the concentration of the surfactant solution and the size of the azobenzene surfactant (Fig. 2). ${ }^{10}$ Such a dependence describes cooperative binding starting from a certain surfactant concentration, in general called critical aggregation concentration (CAC). For concentrations larger than $1 \mathrm{mM}$, the saturation of the thickness increase is reached for all surfactants (Fig. 2).

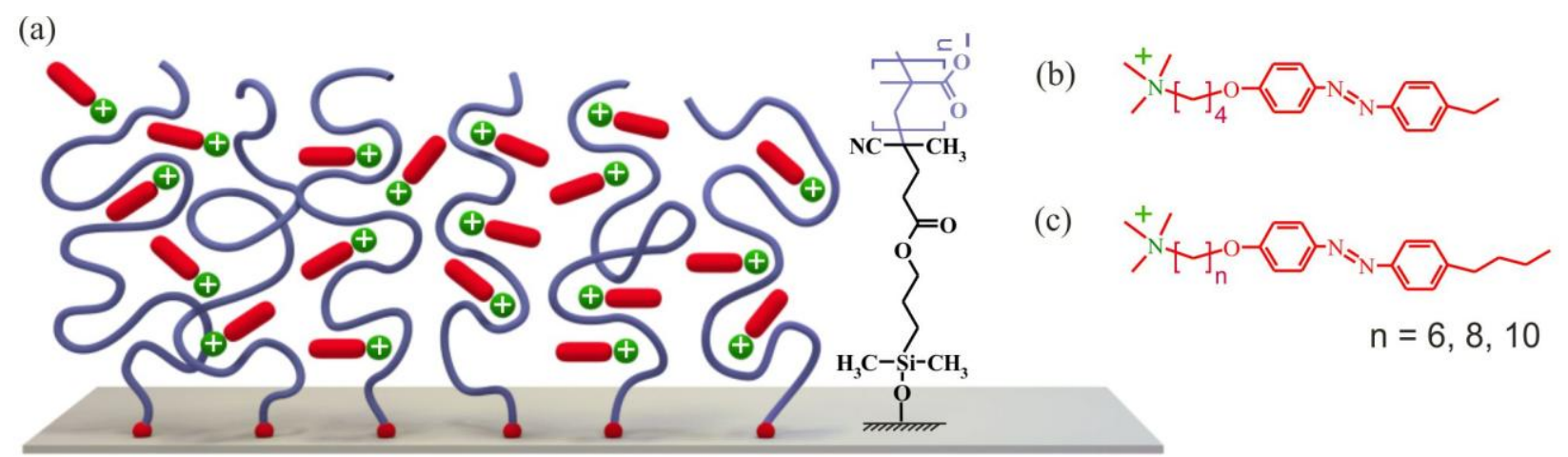

Fig. 1. (a) Chemical structure and scheme of the poly(methacrylic acid) (PMAA) brush loaded with azobenzene-containing cationic surfactants. (b, c) Chemical structure of the surfactants.

As in the case of conventional cationic surfactants, ${ }^{11}$ the alkyl chain length influences the adsorption behavior of the azobenzene surfactant inside the polymer brush. The longer the hydrophobic spacer between the azobenzene group and the charged head, the larger is the increase in the thickness of the polyelectrolyte brush. When calculating the degree of binding $(\beta)$ from the data in the plot presented in Fig. 2 (calculation described in section Methods and Materials), one finds that the maximally achievable degree of binding in studied brush for all four surfactants is in the range of $\beta=1.1 \pm 0.2$ (see Supporting Information, Fig. S3). For the case of the shortest surfactant $\left(4 \mathrm{CH}_{2}\right)$ the onset of the binding to the brush occurs at larger concentrations due to weaker interactions between the polymer chains and the surfactants. ${ }^{48}$ 
The degree of binding $(\beta)$ of the azobenzene containing surfactant to the PMAA brush depends strongly on the PMAA grafting density, similar to conventional surfactants. ${ }^{11} \beta$ drops from 1.0 to 0.55 with increasing graft density from 0.02 to $0.1 \mu \mathrm{mol} / \mathrm{m}^{2}$ as measured for surfactant containing $6 \mathrm{CH}_{2}$ groups in the hydrophobic spacer (see Supporting Information, Fig. S4). The concentration of the surfactant solution in which the brushes were incubated was $0.45 \mathrm{mM}$, a slightly lower value than the critical micelle concentration $(\mathrm{CMC})$ of the surfactant $\left(\mathrm{CMC}=0.5 \mathrm{mM}\right.$ in the case of $6 \mathrm{CH}_{2}$ spacer). ${ }^{23}$

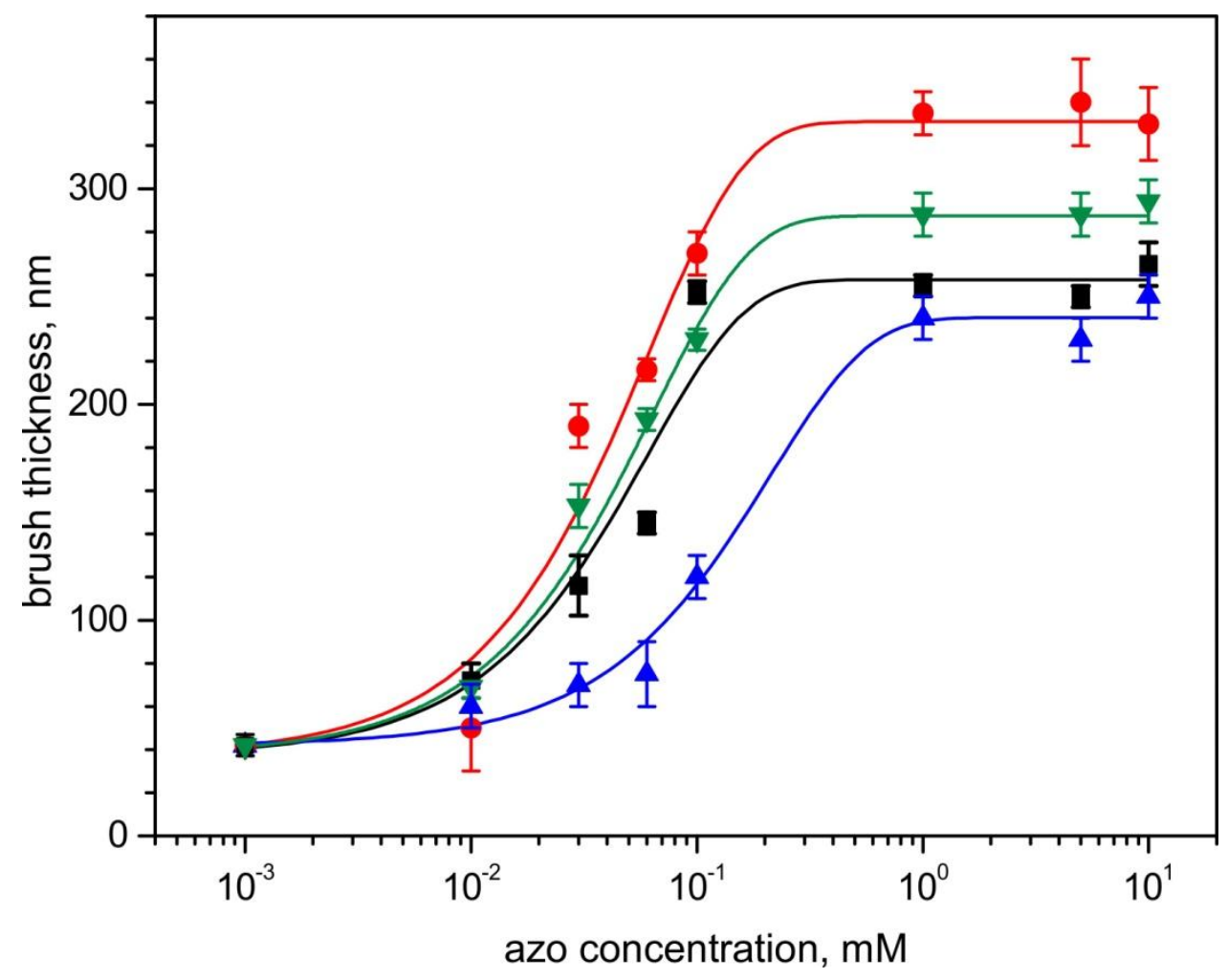

Fig. 2. Dependence of the dry brush thickness on the concentration of the surfactant solution to which the brush was exposed. Four types of surfactants differing in the length of methylene spacer are employed: $4 \mathrm{CH}_{2}$ (triangles, blue line), $6 \mathrm{CH}_{2}$ (squares, black line), $8 \mathrm{CH}_{2}$ (inversed triangles, green line), $10 \mathrm{CH}_{2}$ (spheres, red line).

Within the brush, however, the surfactant molecules are present in the aggregated state as it can be seen from the hypsochromic shift of the UV absorption peak in a trans-conformation from $353 \mathrm{~nm}$ in solution to $335 \mathrm{~nm}$ in the brush (see Supporting Information, Fig. S5). It is well known that azobenzenes form $\mathrm{H}$-aggregates in solution at high concentrations accompanied by a blue shift of the absorption maximum 
of the trans isomer in the UV-visible spectrum. ${ }^{49}$ The large blue shift $(18 \mathrm{~nm})$ is a clear indication of the formation of large aggregates, in which the surfactant molecules can either be oriented tail-to-tail or tailto-head.

The existence of the aggregates within the brush does not influence the roughness of the film (at least just after incubation). A significant increase in the roughness (up to $10 \mathrm{~nm}$ ) is observed under irradiation with homogeneous UV light of 365 nm wavelength (see Supporting Information, Fig. S6). The change in thickness and roughness of the polymer brush under homogeneous irradiatation is outside the scope of the present study and will be reported elsewhere. ${ }^{50}$

Fig. 3h shows the film topography of the photosensitive polymer brushes loaded with the azobenzene containing the azobenzene surfactant $\left(6 \mathrm{CH}_{2}\right.$ spacer $)$ immediately after preparation. The surface is atomically smooth with a roughness of $0.5 \mathrm{~nm}$ over an area of $10 \mu \mathrm{m}$ x $10 \mu \mathrm{m}$. The roughness does not depend on the amount of the adsorbed molecules for all surfactants studied. We stress that after exposing the brush to surfactant solutions of different concentrations the sample is rinsed with water in order to remove the non-bounded surfactants on top of brush surface.

The amount of adsorbed photosensitive surfactants influences strongly the response of the brush topography on irradiation with UV interference patterns. Fig. 3a-c show the topography of the brushes exposed to surfactant concentrations of $0.01,0.06$, and $0.1 \mathrm{mM}$, respectively, after irradiation with an interference pattern at $\lambda=325 \mathrm{~nm}$ during 10 minutes. At small content of the surfactants $(\beta=0.16$, Fig. 3a), the topography does not change significantly, but at larger contents of $\beta=0.53$ (Fig. 3b) and $\beta=$ 1.10 (Fig. 3c) a formation of a characteristic SRG is observed. The periodicity of the SRG is fixed to 1.4 $\mu \mathrm{m}$ being equal to the optical periodicity of the interference pattern, while the SRG height increases with concentration and saturates at $10 \mathrm{~nm}$ (Fig. 3g, black curve). The maximum increase in the SRG height of $10 \mathrm{~nm}$ at an azobenzene concentration of $1 \mathrm{mM}$ (resulting in $\beta=1.1$ ) represents only $4 \%$ change in the total thickness of the photosenstive brush $(\mathrm{h}=250 \mathrm{~nm}$, see Fig. 2, black line). Further 
irradiation does not lead to an increase in the SRG height. This behaviour could be explained by considering the kinetics of trans-cis photo- isomerization reaction of the surfactant molecules. It is known that irradiation induced reorientation of the azobenzene units perpendicularly to the polarization of the incident light is one of the driving mechanisms of the mass transport within the polymer film, eventually leading to the SRG formation. ${ }^{51}$ During irradiation, multiple photo-isomerizations from trans- to cis- and back should occur in order to induce the reorientation of the azobenzene groups. However, the photo-isomerization of surfactants appears only as a single event during irradiation, implying moderate changes in orientation of the azobenzene molecules. Indeed, we have found that in the PMAA brush/azobenzene surfactant complexes there is no preferred orientation when irradiating with polarized light of homogeneous intensity. The equilibrium in-plane isotropic state is immediately achieved within the brush after irradiation is stopped. Thus, the change in brush topography is most likely induced by a change in thickness of the irradiated areas, rather than mass transport, as it is known from physisorbed photosensitive films. ${ }^{52}$

Although, during irradiation the height of the inscribed pattern could not be increased to more than few percent of the total brush thickness, significant changes in the height of the SRG are achieved after exposing the irradiated samples to a good solvent, i.e., DMF. Fig. 3d-f depict the same area on the brush topography as shown in the Fig. 3a-c after subsequent treatment of the surfaces with the DMF. The SRG height increases from 50 to $150 \mathrm{~nm}$ and finally to $240 \mathrm{~nm}$ for the concentrations of $0.01,0.06$, and $0.1 \mathrm{mM}$, respectively. In the latter case, the SRG height nearly coincides with the total height of the photosensitive brush. The explanation of this phenomenon is the opto-mechanical scission of the polymer chains induced locally in the areas of the maximal opto-mechanical stresses. During irradiation a certain number of polymer chains ruptures and the ruptured chains are removed from the substrate during rinsing with a good solvent. In the case of a small amount of surfactant molecules (Fig. 3d-e) it is not clear whether the rupturing occurs near the surface or at an arbitrary point along the polymer 
chain. If no material were left in between the grating, it would indicate that all chains rupture at the brush/surface interface. Since some amount of polymer remains between the topography maxima (Fig. 3d-e), these areas could either be composed of short chains of the same grafting density as the original brush, or of longer chains of lower density, or a mixture thereof. However, when the surfactants is present in large amounts $(\beta=1.1$, Fig. 3f) the rupturing does occur near the solid surface. Indeed, the height of the remaining SRG is nearly equal to the total height of the brush loaded with the surfactants. Concurrently, only a very thin organic layer (1-5 nm in thickness) is detected between the remaining brush stripes.
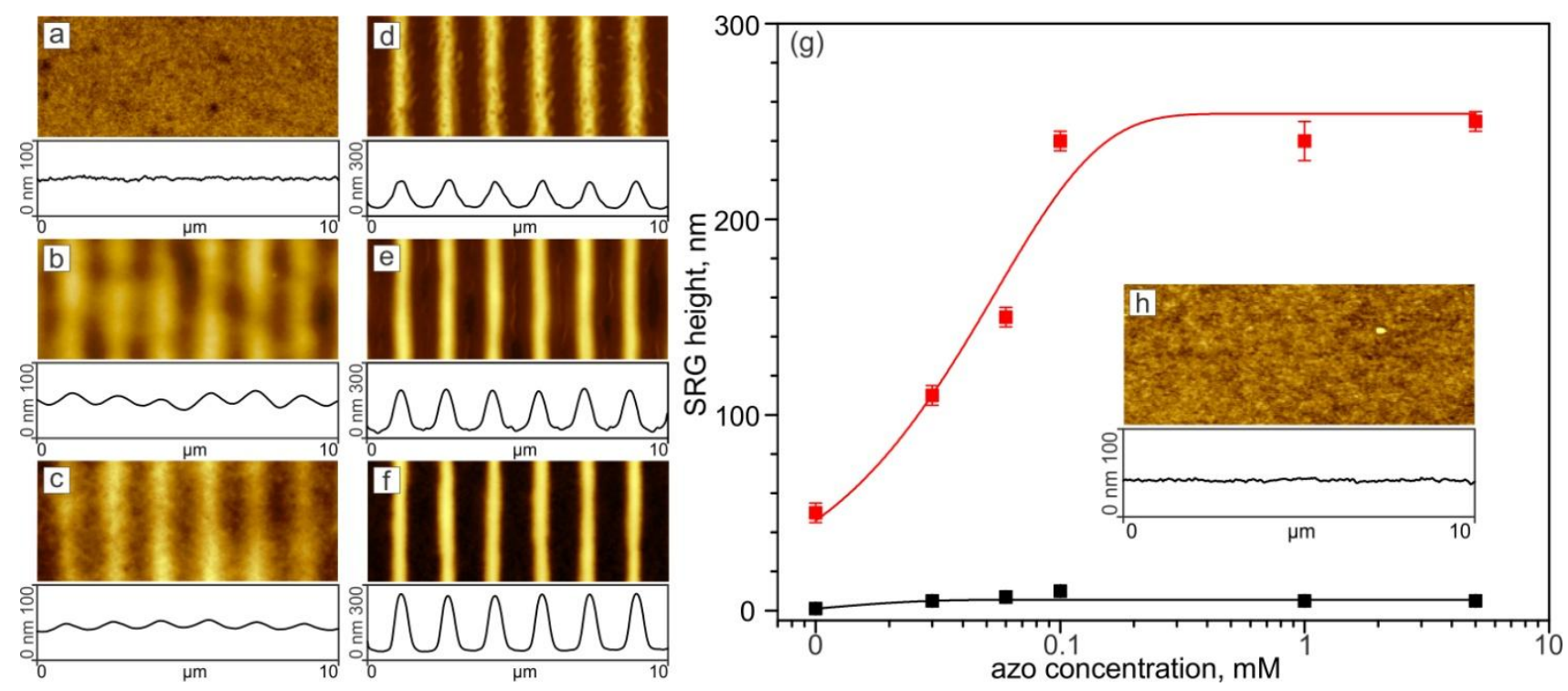

Fig. 3. $(a, b, c)$ AFM micrographs of the PMAA brush loaded with azobenzene-containing surfactant (spacer length: $\left.6 \mathrm{CH}_{2}\right)$ after irradiation with an UV interference pattern $(\lambda=325 \mathrm{~nm}$, SRG periodicity $=$ $1.4 \mu \mathrm{m}$ ) for three different concentrations of the surfactant: 0.01 (a), 0.06 (b), $0.1 \mathrm{mM}$ (c), respectively. (d, e, f) Corresponding AFM micrographs of samples a-c after subsequent exposure to DMF. (g) Dependence of the SRG height on azobenzene concentration immediately after irradiation (black curve), and after subsequent treatment with DMF (red curve). (h) AFM micrograph of the PMAA brush loaded with $6 \mathrm{CH}_{2}$ surfactant before irradiation.

A similar behavior, at which polymer chains can rupture the brush/surface interface during the SRG formation, has been reported for the case of covalently attached azobenzene groups (Disperse Red 1 (DR1)) to tethered polymer chains. ${ }^{38,39}$ There, we did not find a complete removal of the local brush 
material, even at the maximum possible load with azobenzenes: only $75 \%$ of the chains were found to rupture. A complete removal of the active PMAA block from PMMA-PMAA diblock-copolymer brush attached to the solid surface through PMMA block was reported for the loaded with photosensitive surfactants. ${ }^{21}$ At maximum degree of loading $(\beta=0.8)$ complete scission was achieved for an irradiation dose of $\mathrm{J}=2.3 \mathrm{~J} / \mathrm{cm}^{2}$. In the case of a PMAA homopolymer brush, the irradiation dose was $\mathrm{J}=12.4$ $\mathrm{J} / \mathrm{cm}^{2}$. This difference might be explained by the fact that the grafting density of the PMMA-PMAA diblock-copolymer brush, $\sigma_{P M M A-P M A A}=0.4 \mathrm{~nm}^{-2}$, was more than an order of magnitude larger than in the case of the PMAA brush, $\sigma_{P M A A}=0.01 \mathrm{~nm}^{-2}$. This implies larger tension within the tethered polymer chain and thus a smaller force might be required to rupture a chain. ${ }^{44}$
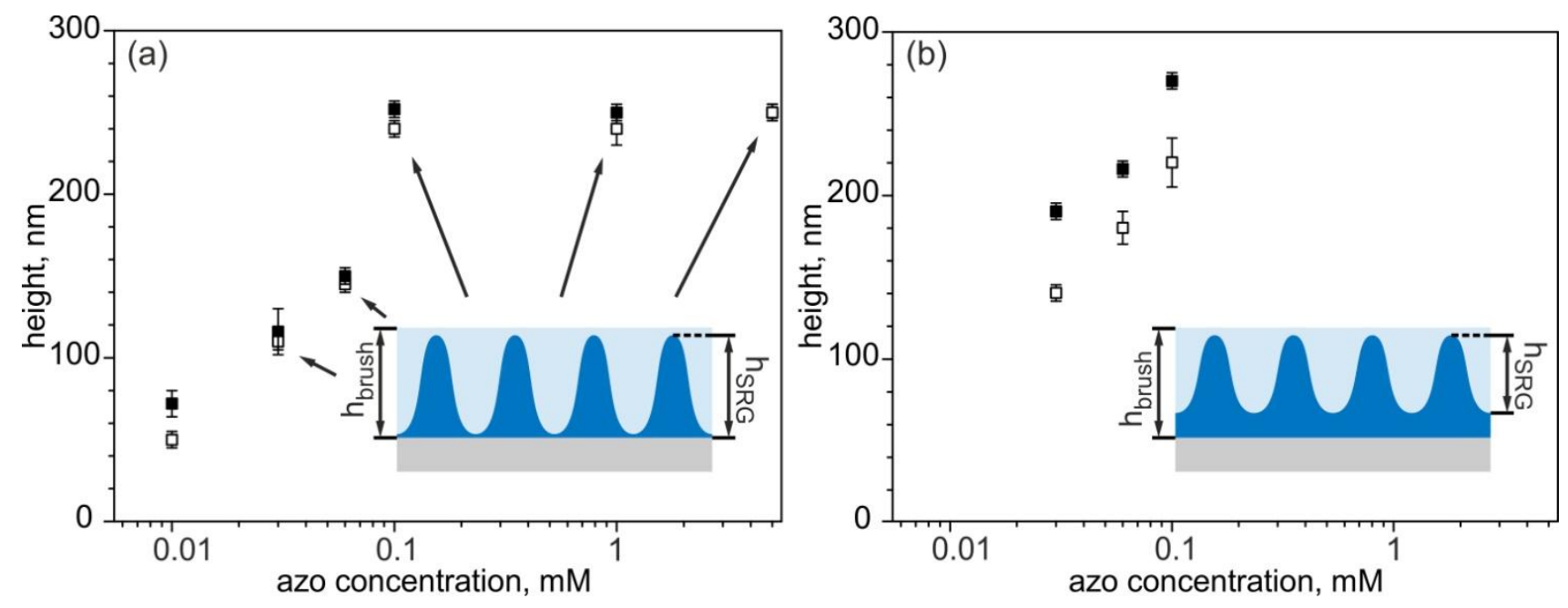

Fig. 4. Dependence of the SRG height of polymer brushes loaded with azo- $6 \mathrm{CH}_{2}-\mathrm{TMAB}$ (a) and azo$10 \mathrm{CH}_{2}-\mathrm{TMAB}(\mathrm{b})$ on the concentration of the photosensitive surfactants. Black squares show the thickness of the brush after loading the PMAA brush with surfactants, open squares show the SRG height after exposure of irradiated PMAA/azobenzene complex brush to a good solvent. Schemes of the SRG profile in brushes are provided on both plots to illustrate distribution of the polymer material.

At these irradiation conditions, in the case of shorter surfactant (azo- $6 \mathrm{CH}_{2}-\mathrm{TMAB}$, Fig. 4a) it was possible to completely remove the brush material locally starting from the concentrations of $0.1 \mathrm{mM}$ $(\beta=1.1)$. In the case of longer surfactants (azo- $8 \mathrm{CH}_{2}-\mathrm{TMAB}$, azo- $10 \mathrm{CH}_{2}-\mathrm{TMAB}, \mathbf{F i g}$. $\left.4 \mathbf{b}\right)$ there is always some material left between the topography maxima (see scheme in Fig. $\mathbf{4 b}$ and Fig. 5). This can be explained by considering that "bulky" molecules or objects cannot penetrate deep into the brush, as 
reported previously. ${ }^{18,53,54,55}$ This implies that the brush is not homogeneously functionalized with photosensitive moieties in the direction perpendicular to the plane of the film. In our previous paper we have shown in using PMMA-PMAA diblock-copolymer brushes that the opto-mechanical stress generated within the film during irradiation cannot induce rupturing of the inert block (PMMA) not functionalized with photosensitive groups.

To illustrate this phenomenon we plot the brush height (circles) and that of the SRG (squares) as a function of the surfactant length in Fig. 5. For these samples the concentration of the surfactants was chosen in such a way that the degree of binding for all surfactants $\left(4 \mathrm{CH}_{2}, 6 \mathrm{CH}_{2}, 8 \mathrm{CH}_{2}, 10 \mathrm{CH}_{2}\right)$ is 1.0 . In the case of longer surfactants $\left(8 \mathrm{CH}_{2}, 10 \mathrm{CH}_{2}\right)$ the $\mathrm{SRG}$ height is 180 and $220 \mathrm{~nm}$ at a total brush height of 230 and $270 \mathrm{~nm}$, respectively. For the shorter surfactant (i.e., $6 \mathrm{CH}_{2}$ ), the SRG and brush height coincide within the range of experimental error, indicating that in between the topography maxima there is almost no brush material left.
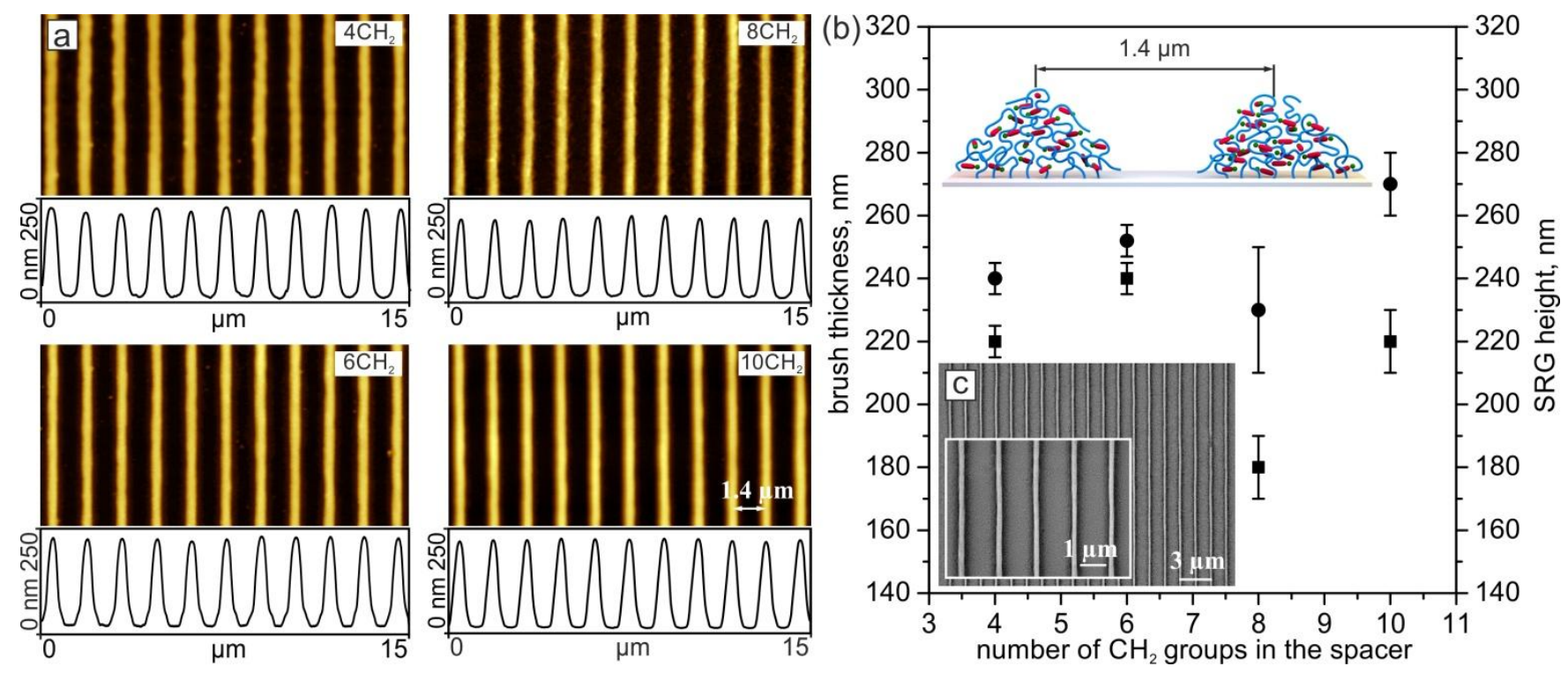

Fig. 5. Dependence of the brush (circles) and SRG (squares) height on the length of the hydrophobic spacer connecting azobenzene unit and charged head group (b). The upper inset shows a scheme of the brush shape. Corresponding AFM micrographs are shown in (a) for surfactants containing 4, 6, 8, and $10 \mathrm{CH}_{2}$ spacers. (c) SEM micrograph of the structured polymer brush showing nano-stripes of tethered chains. 
In the case of the shortest surfactant (i.e., $4 \mathrm{CH}_{2}$ ), it was not possible to achieve a complete removal of brush material from the irradiated areas although on account of the ease of surfactant penetration deep into the brush, the results should even be more pronounced than for the surfactant with a $6 \mathrm{CH}_{2}$ spacer. At this point, we can only hypothesize about the actual reason for this behavior, which has nevertheless been reproduced a number of times. A probable explanation involves the interaction potential of the surfactant with the brush. A shorter spacer also means reduced hydrophobicity, and thus an overall reduced interaction strength with the brush. That is, although the forces that can potentially be generated during trans-cis isomerization, do not couple strongly enough with the polymers in order to transmit or sustain the forces generated within the brush.

The shape of structured polymer brushes resembles "bushes" consisting of polyelectrolyte chains loaded with photosensitive surfactant, extending as nano-stripes across the surface (see scheme in Fig. 5). These nano-bushes are still photosensitive as one can see from the following experiment (Fig. 6). The structured brush (loaded with $6 \mathrm{CH}_{2}$ surfactants) was irradiated for the second time under identical conditions (i.e., $\lambda=325 \mathrm{~nm}, \mathrm{I}=5 \mathrm{~mW} / \mathrm{m}^{2}, \mathrm{D}=1.4 \mu \mathrm{m}$ ) and subsequently washed once with DMF, but with the intensity pattern oriented perpendicularly to the first one (cross-irradiation). After this procedure an array of round spots arranged in a square grid consisting of tethered polymer chains was generated (Fig. 6 a, c). The diameter of these spots is $360 \pm 20 \mathrm{~nm}$, and the height is $340 \pm 10 \mathrm{~nm}$, which corresponds to the height of the non-irradiated brush. A thin layer of brush material along the nano-stripes of $\mathrm{h}=8 \pm 2 \mathrm{~nm}$ is left after cross-irradiation. Although the results presented in Fig. 6 are similar for all brushes investigated, as the most striking example we show the brush with larger grafting density $\left(\sigma=0.02 \mathrm{~nm}^{-2}\right)$, and thus larger thickness and correspondingly increased SRG height. The crossirradiation can also be conducted at any angle to the primary nano-stripes in order to generate nanospots with a different shape, e.g., ellipses. For instance, cross-irradiation at $45^{\circ}$ results in the formation of elongated brush nano-spots ordered hexagonally (Fig. $6 \mathbf{b}, \mathbf{d}$ ). The major and minor diameter of the 
ellipses, its height, and periodicity are $510 \pm 10,340 \pm 10,340 \pm 10 \mathrm{~nm}$, and $1.4 \mu \mathrm{m}$, respectively. Similar results are observed for all photosensitive surfactants studied here; the only difference being that in between the nano-stripes or nano-spots there is always brush material left. This further indicates that larger surfactants do not tend to penetrate deep into the brush.
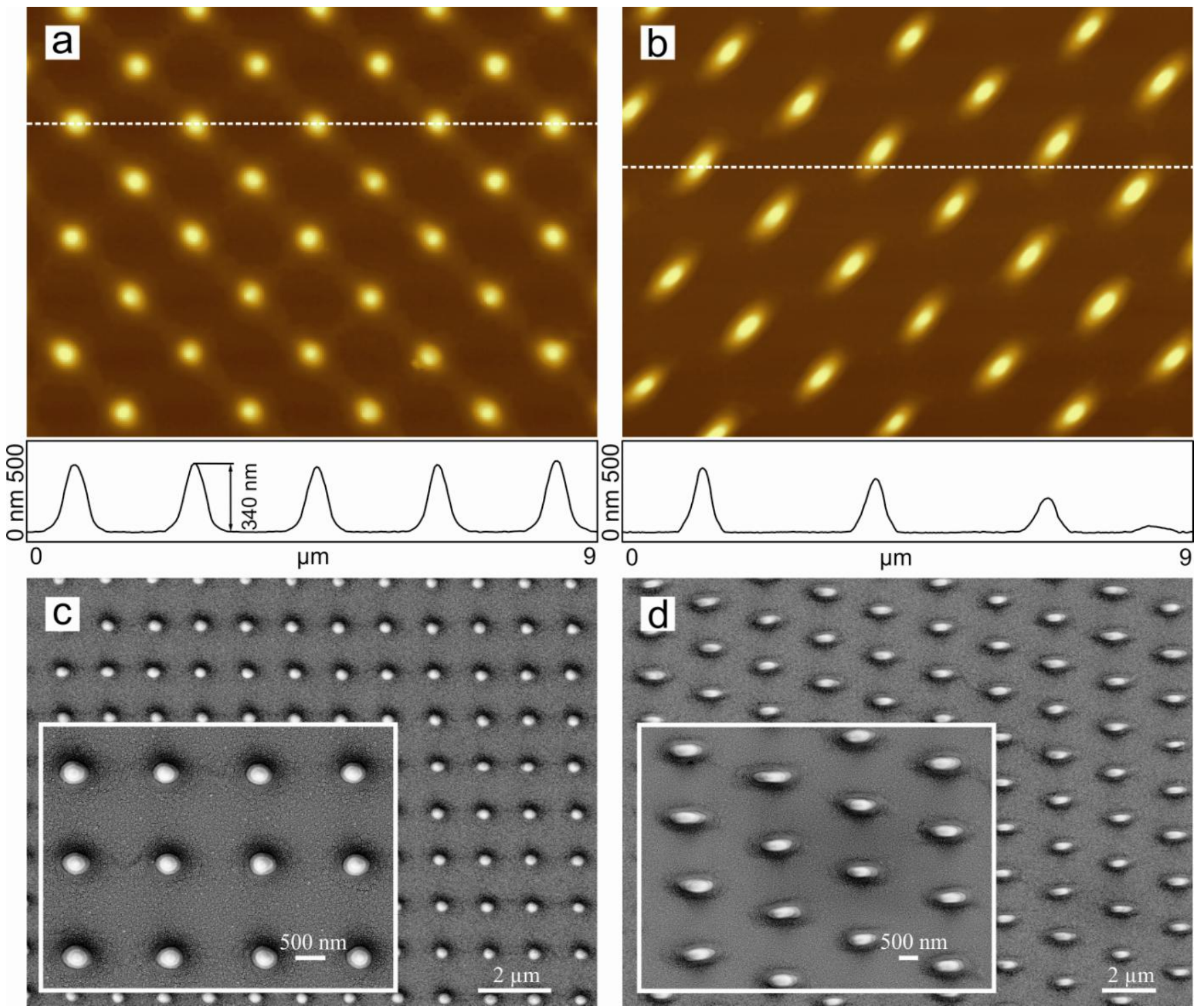

Fig. 6. (a, b) AFM micrographs of the photosensitive brush topography after cross-irradiation and exposure to a good solvent (DMF). (c, d) Corresponding SEM micrographs. The irradiation conditions were varied between $90^{\circ}$ and $45^{\circ}$ of cross irradiation.

After the structure generation is completed, one can partially remove the remaining surfactants from the brush by exposing in water for a certain time, i.e., the nano-structured brush is again ready for further 
treatment and refinement. The removal of the surfactants from the brush is detected experimentally by measuring the thickness of the dry brush after washing the specimens with water (Supporting Information, Fig. S7). In short, the brush was first loaded with surfactants and characterized by AFM. Afterwards, it was exposed to water for 20 minutes followed by drying and measurement of its thickness. This procedure was repeated multiple times. We have found that ca. $66 \%$ of the surfactants are washed out within 160 hours resulting in a drop of the binding constant from 0.55 to 0.18 . It was reported that a conventional surfactant (DDTAC) with $12 \mathrm{CH}_{2}$ groups in a hydrophobic tail binds strongly to a polyelectrolyte brush and cannot be removed by expose in water. ${ }^{11}$ In our case, although the surfactant has a comparable length of hydrophobic tail, it bears photosensitivity due incorporated azobenzene group. Since the exposure to water was conducted under day light, at least $12 \%$ of all surfactant molecules adsorbed in the brush are in the cis-state. ${ }^{28}$ The cis-isomer being more hydrophilic was shown desorbs easily out of negatively charged soft objects such as for instance microgels. ${ }^{26}$ This $^{2}$ can be a possible mechanism for the removal of the azo-surfactant from the brush.

In summary, our approach represents a new way of structuring polymer brushes on a nanometer length scale with a rather soft intervention, since the irradiation intensities are moderate. It can also be used as a method to refine the structure of electrolyte brush that had been prepared by other means.

\section{Conclusions}

Photosensitive polymer brushes were prepared by coupling PMAA tethered polymer chains with cationic azobenzene containing surfactants. The PMAA brush was first grown using the "grafting from free radical polymerization" from flat, silica-based surfaces and supramolecular complexes between the negatively charged brush and four different cationic surfactants were formed by exposing the brushes to solutions of the azobenzene surfactant. The surfactants differ in the length of the hydrophobic spacer connecting the cationic head group and the azobenzene unit. In this study the spacer length was varied 
between 4 and $10 \mathrm{CH}_{2}$ groups. The amount of the surfactants within the brush depends on the concentration of the solution to which the brush was exposed to and the size of the surfactant. The maximal degree of binding for all four surfactants was found to be ca. $\beta=1.1 \pm 0.2$. We have shown that the polymer brush loaded with photosensitive molecules responds strongly to the irradiation with light. In the case of a homogeneous intensity distribution but alternating wave lengths of the incoming light, one can switch the roughness of the brush between $0.5 \mathrm{~nm}$ and $10 \mathrm{~nm}$, periodically. Interesting effects can be obtained by irradiating the brush with interference patterns with spatially varying intensity distributions. The brush show a similar behavior as had been previously reported, ${ }^{21}$ the presence of the photosensitive surfactant leads to the generation of strong opto-mechanical stresses within the brush that are localized in areas of maximal intensity. This leads to rupturing of the tethered polymer chains, which, in turn, can be removed to leave a structured polymer brush behind, the shape of which can be varied depending on the irradiation pattern. Out of the four studied surfactants only one with an intermediate length (i.e., $6 \mathrm{CH}_{2}$ ) of the hydrophobic spacer connecting the cationic head with the hydrophobic tail could produces a significant rupturing of polymer chains up to the complete removal of a single chain off the substrate. This corresponds to local irradiation conditions. Larger surfactants do not penetrate deep enough into the brush; consequently, after rupturing a left over layer of polymer material remains on the substrate. As the surfactants are not covalently bonded to the polymer, they can be partially washed out by treating the brush with water. This nano-structured brush can then be reused for further functionalization.

ACKNOWLEDGMENTS: This research is supported by the VolkswagenStiftung, Germany; DFG (SA1657/8-1); International Max Plan Research Graduate School on Multiscale Bio-systems (Potsdam, Germany) and Helmholtz Graduate School on Macromolecular Bioscience (Teltow, Germany). The work at NC State was supported by the National Science Foundation (Grant no. DMR-1404639). 